\title{
"BOZALENGO", ESTA BOCA NO ES MIA: LA VOZ DEL SUJETO POPULAR NEGRO EN LOS TESTIMONIOS (LATINO) AMERICANOS Y CARIBENOS
}

\author{
“BOZALENGO", ESTA BOCA NÃO É MINHA: A VOZ DO SUJEITO POPULAR \\ NEGRO NOS TESTEMUNHOS (LATINO) AMERICANOS E CARIBENHOS
}

\author{
Victorien Lavou Zoungbo ${ }^{1}$
}

A

La memoria de Stuart Hall, John Beverley, Mis educando-a-s Licence 3 y Masters II, espagnol (2012-2013; 2013-2014)

\begin{abstract}
Resumo: Con el triunfo de la Revolución cubana en 1959, uno de los cometidos políticos de los revolucionarios era propugnar una cultura antiburguesa y antiimperialista. La práctica institucionalizada del testimonio constituía entonces uno de los espacios donde se fraguaba esta "nueva cultura". Héroes populares, entre los cuales negros, tenían de esta manera acceso a la autoridad de la palabra escrita. Pero ¿qué significa realmente, en el caso del sujeto negro popular, "hablar" cuando durante siglos largos fue considerado onto políticamente y por ley como una "pieza" de Indias, un "instrumento útil” ¿Cómo "hablar" desde aquella vorágine cuya impronta sigue marcando las estructuras socio imaginarias de Américas/Caribes? Son unos planteos que contempla este recorrido crítico.
\end{abstract}

Palavras-Chave: Testimonio, "Bozalengo", "Présence-histoire noire", Caribe, Afrodescendientes.

Abstract: Near by the triumph of Cuban revolution on 1959 a some how new cultural practice come to be institutionalized. Popular heroes, among them Black people was then

\footnotetext{
${ }^{1}$ Doutor (Ph.D) em Literatura Latino-Americana pela University of Pittsburgh, USA. Professeur Dr. Université de Perpignan Via Domitia, Perpignan, France. E-mail: lavou@univ-perp.fr
} 
celebrated trought testimony. Is this political shift really mean an access for these heroes to the ordre du discours as M. Foucault would said? Bringing out some contradictions related to testimony as well to the hegemonic word vision in Americas/Carribean, the principal aim of the paper is to recall on how is it possible, for Black people subject, to speak from a faille which deeply still shaping socio historical structures and representativeness in this part of the word.

Keywords: Testimony, «Bozalengo », «Présence-histoire noire », Caribbean, Afrodescents.

Digénesis:

De alguna forma todo empezó con la siguiente invitación benevolente de mi colega Ari Lima:

Prezados Colegas,

Estou como organizador de um número especial da Revista Ponto de Interrogação do Programa de Pós-Graduação em Crítica Cultural da Universidade do Estado da Bahia, sou vice-coordenador do programa atualmente. O tema deste número especial é cultura negra e popular. Pensei em convidá-los a enviar artigos de até 20 páginas (incluindo referencias, notas, etc.), inéditos. Os artigos podem ser enviados em português, inglês, francês ou espanhol. Os artigos devem chegar até 31 de janeiro de 2014. Respondam se têm interesse em participar. Seguem abaixo as normas da revista. Aguardo retorno. Enviem os artigos para o meu e-mail.

Cher collègue,

Je suis touché sincèrement par votre marque d'intérêt pour mes travaux. Bien entendu que je suis intéressé par un tel projet de publication. Juste une chose, qu'entendezvous par «cultura negra e popular »? Peut-on aborder par exemple la manière dont la "ciudad letrada" des Amériques/Caraïbes intègre la voix populaire noire? J'aimerais beaucoup pour ma part examiner cette question à travers la pratique culturelle du "testimonio", en prenant pour exemple Biografía de un cimarrón dont Miguel Barnet a été le «gestor ». J'espère que cela rencontrera vos attentes. Muito obrigado.

Prezado colega, 
De fato tenho muito interesse sim em conhecer e divulgar seu trabalho. Vi seu currículo, não pude ler ainda com a devida atenção o material que enviou para mim, mas fiz uma leitura dinâmica e me impressiona o fato de que reflete sobre a questão do negro, sobre cultura negra em uma perspectiva interdisciplinar. Do mesmo modo, me impressionou o fato de que formula questões importantes que vão além do debate sobre o lugar de vítima ou mesmo sobre a mera validação de estratégias de enfrentamento da colonização, da escravidão, do racismo. Me parece que reflete também sobre o negro na condição de agente, sujeito de suas derrotas e fracassos, assim como aponta para os desafios contemporâneos seja no continente africano ou na diáspora.

Neste sentido, minha compreensão sobre cultura popular e negra passa pela afirmação de que existem dimensões, aspectos das tradições e práticas culturais elaboradas pelos negros que remetem ou mesmo inscrevem histórica e socialmente uma diferença em relação a outros grupos raciais. A meu ver esta diferença se definiu ou se define pela experiência da colonização européia, pela escravidão, pela memória de África na diáspora, pelo racismo, por perspectivas de "embranquecimento" ou "enegrecimento". Além disso, em muitos estados-nação afro-diaspóricos, como é o caso do Brasil, se define, por um lado, o popular como o negro e aquilo que remete a ele: a pobreza, um jeito de ser brasileiro, símbolos como a mulata, o samba ou o futebol. Porém, ao mesmo tempo se nega ou se interdita a presença e a representação do negro nos espaços de poder, prestígio e visibilidade social ampla como é o caso dos meios de comunicação, das instituições públicas que controlam o estado nacional ou naquelas privadas que produzem e acumulam riqueza material. É como se houvesse uma esquizofrenia simbólica e cultural. Logo, sua proposta de abordagem da temática que propus ("... la manière dont la "ciudad letrada" des Amérique/Caraïbes intègre la voix populaire noire") é ótima.

Espero que tenha sido claro.

Esta breve respuesta que dio Ari Lima terminó de convencerme y decidí entonces ser partícipe del proyecto que el ideó. Ahora bien, al contrario, tal vez, de los demás participantes, no me centraré en el examen o en la recepción de tal o cual forma cultural (auto) identificada como negra y popular (HALL, 1992:21-33); me ocuparé más bien brevemente de la manera cómo la ciudad letrada americana/caribeña (desde luego nada homogénea) pretendió integrar, reconocer, ratificar o dar lugar a la voz popular negra. Partiré entonces de un caso que por muy limitado que parezca no es menos significativo o llamativo, esto es la emergencia e institucionalización de la práctica cultural del testimonio popular, sobre todo, a postrimerías del triunfo de la Revolución cubana en 1959.

En este recorrido crítico que considero ante todo como una relatoría, me benefician los cursos y seminarios que vengo dando a los alumnos de Licenciatura (Licence 3) y de postgra- 
do (Master II recherche) de mi universidad sobre el "testimonio popular" en Américas/Caribes. Las reacciones diversas y a veces francamente encontradas de mi alumnado ante mis planteos remiten, bien que sean formuladas con llaneza y algo de ingenuidad, a las interrogaciones epistemológicas, a los debates hermenéuticos y políticos habidos (y siempre actuales) en torno a la práctica del testimonio popular en estos lugares del mundo (BEVERLEY y ACHUGAR, 2002; ARONNA, 2008:162-187).

Sin ser totalmente una novedad en Américas/Caribes, se trataba esta vez, con la Revolución cubana mediante pero también en un ambiente general transcontinental y mundial de cambios y transformaciones políticos profundos, de fomentar/asentar una forma cultural disidente que cuestione la literatura culta burguesa dominante que, por ejemplo, si bien es cierto que no descartaba totalmente la "présence-histoire" (ZOUNGBO, 2007) negra, tampoco la consideraba una preocupación central, digna de interés aunque sea porque está incuestionablemente ligada a la "invención" de Américas/Caribes. Una insensatez que, hasta cierto punto, los revolucionarios y los "intelectuales solidarios" querían subvertir, contrarrestar o desplazar haciendo hinca pie en formas culturales (cines, artes, literaturas, periodismos, bailes, etc.) populares, o sea en formas culturales que, según se creía, eran capaces de movilizar a la mayoría con motivo de la emergencia del "hombre nuevo"; un sujeto que al final no esté enajenado por ciertos atavismos nefastos como el racismo, el machismo, el bovarysmo cultural. Por otra parte, este "hombre nuevo" habría de ser también nacionalista cuando no revolucionario y antiimperialista.

En este sentido, el testimonio popular venía entonces a ser uno de los espacios donde iba a fraguarse una contracultura comprometida con luchas menores, con voces y experiencias despreciadas, ninguneadas, ilegitimizadas. En el caso de la Cuba revolucionaria, se procuraba oficialmente, desde unos requisitos establecidos previamente, recoger y difundir (a través de su publicación por la editorial Casa de las Américas) aquellas voces y experiencias subalternas tenidas a menos por las historiografías ancien régime por así decirlo pronto. Con todo ello, se buscaba granjear legitimidad popular y se quería consolidar la nación cubana desde bases culturales nuevas, desde valores emancipadores.

Ahora bien, esa opción prioritaria por la voz de los de abajo, por los sin voces no está sin embargo exenta de contradicciones puesto que, es de recordarlo, se daba en una forma- 
Pontos de Interrogação, v. 3, n. 2, jul./dez. 2013

Revista do Programa de Pós-Graduação em Crítica Cultural

Universidade do Estado da Bahia (UNEB), Campus II - Alagoinhas - BA

ción socio histórica e intelectual postcoloniales y postesclavistas. Esto supone necesariamente unas herencias o legados paradojales (muchas veces denegadas), una jerarquización cultural naturalizada, un inter dicta, unas reactivaciones, no siempre conscientes, de preconceitos arraigados y por tanto difíciles de desvelar y desmontar. No ayudaba tampoco que los revolucionarios, desde los inicios, se mostrasen poco propensos a dar un lugar público consolidado a las memorias de los agravios coloniales y esclavistas cuyas consecuencias seguían vivas. Lo cual, pese a las medidas positivas que ellos tomaron, dio lugar a debates y controversias porque entonces los principios rectores de la Revolución no necesariamente cazaban con algunas vivencias y creencias populares negras (bailes, organizaciones sociales y religiosas, vestimentas, memorias africanas, culto a los dioses africanos, etc.). Ni que se hablen de las memorias dolorosas de la mal llamada "guerrita del 12" que arrasó con miles de negros que, dando seguimiento al llamado de los líderes del Partido Independiente de Color (PIC), reclamaban mayor consideración como ciudadanos cubanos, equidad y justicia social. Los negros cubanos resentían tanto más las vejaciones sociales duraderas que sufrían cuanto que su participación abrumadora en las guerras anticoloniales y por la independencia de Cuba no redundó en su aceptación como ciudadanos plenos. Por otra parte, la impresión general Ni los cambios importantes habidos en los años 40 (avances constitucionales por ejemplo) en la isla no llegaron a cambiar fundamentalmente la situación de la inmensa mayoría de los negros en la isla. Tampoco, según los imaginarios colectivos imperantes, los negros casaban con los "ideales de la raza cubana” imaginada (ZOUNGBO, 2009). Todo parecía indicar por lo demás que el "miedo al negro" (un potente y activo constructo político e imaginario) no se limitaba al siglo XIXe. ¿Cómo entonces en estas circunstancias prestar debida atención a la Revolución y a sus promesas de "nuevos tiempos" venideros si al mismo ella reprimía las prácticas culturales con las cuales se identificaban los propios negros en su inmensa mayoría?

A lo que antecede (desde luego opinable según las "bibliotecas" cubanas y las cegueras intelectuales) y que se ha reseñado a penas, habría que tomar en cuenta el interrogante siguiente que, hasta donde yo sepa, se ha quedado rezagado por ser muy poco contemplado o examinado por los mayores críticos de la práctica cultural testimonial americana/caribeña. En efecto, si uno se remite al Code noir francés (1685) y a otros códigos negreros que le son afines o que se inspiraron en él, es dable preguntarse ¿Qué significa exactamente para el sujeto 
negro africano "hablar" cuando durante siglos largos ha sido identificado onto políticamente a "pieza" o "leña" de Indias, a "instrumento útil"?

De modo que, a mi parecer, la muy saludable ruptura política inaugurada por la Revolución cubana, no necesariamente no merma ni socava este interrogante. Muy al contrario cobra validez e interés crítico cuando uno se detiene realmente en una de las instancias de producción/difusión del testimonio popular. Me refiero aquí al espacio que comparten, desde lugares de enunciación dispares, desde temporalidades divergentes, el sujeto/testigo-testimoniante (en muchos casos analfabeta o ileterado) y el sujeto/gestor (por lo general antropólogo, "intelectual solidario", parte de le "cuidad letrada", etc.).

$\mathrm{Al}$ acuñar el neologismo de "bozalengo" pretendo justamente insistir en el ninguneo estructural e imaginaria persistente y activo de la voz negra por las prácticas hegemónicas americanas y caribeñas. Conviene ahora detenernos en él para deletrearlo mínimanente, dando así a entender la capacidad de lectura crítica, a contrapelo, que brinda o abriga para quienes se interesan por el "hablar" de los negros tanto en Américas/Caribes como en sus diásporas regionales, transregionales y transcontinentales.

"Bozalengo" combina dos términos: "Abolengo" y "Bozal". Ambos remiten a mapas mentales, a hábitos socio imaginarios aristocratizantes (caso del primero) y la violencia fundadora de la gramática colonial y esclavista en Américas/Caribes. El primer término presupone un lazo irrefragable entre un apellido y la tierra que en la España feudal se trasmitía de padres a los primogénitos/varones. Era entonces una estructura patriarcal que programaba y naturalizaba la exclusión radical de las hijas/hembras del acceso a la propiedad, al nombre del padre (no así forzosamente de los segundones o de los tercerones/varones). Era al mismo tiempo una manera de ratificar la exclusión de las mujeres de la palabra rectora, del discurso social vertebrador puesto que la posibilidad (reconocida y socialmente garantizada) de "hablar" dependía del valerse del orden socialmente codificado de una genealogía (imaginada ilustre, pura e inmemorial), de los haberes que uno tuviese o de las hazañas guerreras que uno realizase.

"Bozal" es una categoría de siniestra memoria en las sociedades poscoloniales y post esclavistas. ¿Qué era? (la única pregunta que valga en este caso). En primer lugar, era la otra cara del negro crioullo o criollo esclavizado. El "bozal" era un africano negro esclavizado 
Pontos de Interrogação, v. 3, n. 2, jul./dez. 2013

Revista do Programa de Pós-Graduação em Crítica Cultural

Universidade do Estado da Bahia (UNEB), Campus II - Alagoinhas - BA

recién arribado, en los barcos negreros, a las Américas/Caribes, después de haber sufrido en carne propia los horrores de la travesía transatlántica (REDIKER, 2013). Él no nació ni se crió (como por ejemplo en el caso de los esclavizados jóvenes trasegados a las Américas/Caribes) en las tierras americanas y tampoco era afín a las culturas y sobre todo a los idiomas de los esclavistas (francés, inglés, español, portugués, holandés, etc.) que estaban en trance de consolidación. Por falta de una gramática unificadora, por las procedencias diversas de los colonos y sobre todo por el nivel de la educación formal que la mayoría de ellos tenía, lo que tampoco les capacitaba para "hablar" latines. En efecto, muchos de ellos eran analfabetos o semiletrados. Lo que hace que despecho del "capital racial" que les procuraba y garantizaba la "gramática colonial esclavista", padecían la "violence de la lettre", la violencia de la grafía autorizada que era entonces un puro privilegio reservado a los letrados, a los funcionarios peninsulares, al clero.

"Bozal" remite también a "bozo", un instrumento infernal que en la economía colonial y esclavista se ponía por fuerza a la jeta/boca de los esclavizado-a-s negro-a-s, a la hora de la zafra para que no comieran azúcar y para que no se comunicaran entre sí mientras duraban la faena y contra faena. Dicha prohibición era a la par infamante y mutilante puesto que reiteraba la condición onto política del sujeto negro africano: un esclavizado asimilado a un animal y tratado como tal (el "bozo" se destina a perros y a caballos) que por tanto no estaba autorizado a "hablar" naturalmente.

Los negros africanos esclavizados, esto sí , "recuperaban" su condición ontológica de ser humano (suspendido/"sous rature") y con ella su facultad de "hablar", en las "senzalas"/"bohíos" (para la mayoría de ello-a-s), en las "casas grandes"/“habitations" (para lo-a-s esclavizado-a-s doméstico-a-s o allegado-a-s de una forma u otra), en los espacios de libertad y de subversión del orden colonial esclavista que se conocen como los palenques, los cumbes, los quilombos o los free villages; también les era posible "hablar" cuando los baños o festejos autorizados, obligados o conquistados.

Este habla sin embargo se percibía como una pura jerigonza, un puro ruido, un gruñido, un alarido o una farfulla; en todo caso se retrataba como una irrupción antinatural (y amenazante) en el concierto de la modernidad esclavista y colonial. En pocas palabras, era tenida por un habla de "excepción" que brotaba parigual de un "mercancía"/cuerpo excepcional que, 
por lo demás, solo se destinaba, por ley y derecho naturales, al cumplimiento de trabajos duros en condiciones infrahumanas. Con que, se trataba de un cuerpo que no existía sino para recibir y experimentar maltratos, castigos horrendos y vejaciones extremas.

Ahora bien, la tamaña esquizofrenia estructural en que descansaba aquel orden colonial esclavista daba lugar a cosas que, por ejemplo a ojos inocentes de mis alumnos, parecen raras, descomedidas y sobre todo ilógicas. Muchas veces les tengo que explicar que aquellas rarezas no lo son sino de cara al horror y el asco que nos provocan (y en este sentido ante las indiferencias actuales cuando de la esclavización de los negros africanos se trata, he de confesar y reconocer que la reacción de mi alumnado por más ingenua que parezca es saludable). No eran pues rarezas puesto que el actuar horrendo generalizado de los esclavistas formaba parte de un modus vivendi y operandi refrendado por el sistema colonial esclavista en Américas/Caribes.

Así es que, aun cuando estaba definida jurídica y ontológicamente como "leña" o "pieza" de Indias y que en las interacciones sociales obligadas era percibida como un desecho humano, no era menos acosada, agredida sexualmente por los esclavistas; éstos no dejaban de ejercer sobre ella (y sus hijas, muchas veces engendros/ganado habidos tras las violaciones comunes y corrientes en contra de la "madre-leña") el derecho natural de pernada. Aquella misma "leña" o "pieza" daba de mamar su seno/ubre a la preciosa progenie de los esclavistas blancos (a expensas de los crío-a-s/hijo-a-s de ella) puesto que había que proteger y salvaguardar absolutamente la lozanía y vigor de las mamas de las amas blancas. La "leña" se tornaba en este caso concreto una "vaca lechera" negra. De la misma manera, la alimentación, la higiene íntima (baños), el acicalamiento (vestimenta y peinado, etc.), el goce sexual obligado, corrían a cargo de aquel "excremento" humano, de aquella "pieza". A la "leña", nada más congruente según parece, se le prohibía leer (la Biblia), escribir y educarse hasta entrado el siglo XVIII, dependiendo de las colonias esclavistas.

Un ser humano se veía así impedido, erased, de la condición humana inapelable. Se le reducía entonces, durante siglos largos, a una condición legal y natural de "mercancía" dedicada a la producción de otras mercancías par beneficios ajenos, para beneficios de los mercados europeos, del capitalismo imperial. 
El acceso a las independencias, los "nuevos tiempos" emancipadores poco cambió esa condición jurídica ominosa; tampoco cambiaron las percepciones hegemónicas y populares de los africano negros, de los recién libertos o de los ex esclavizados. No era pues de sorprender que las ciudades letradas americanas/caribeñas no hicieran más que seguir obturando la voz de los sujetos subalternos americanos/caribenos y entre estos mas aun la voz de los negros.

Quienes se destinaban entonces naturalmente a "hablar" en representación de "todos" eran los sujetos blancos criollos que cifraban, en gran parte, su poder de representación (mimética y política) en la "violence de la lettre", en el poder político e imaginario del bolígrafo (constituciones, decretos, periódicos, literaturas y/o ensayos fundacionales, leyes, acuerdos oficiales, etc.) mientras que la inmensa mayoría que ellos pretendían dirigir, gobernar y encauzar eran analfabetos y, según la visión global entre las élites, quedaban patológicamente "enfrascadas" en la oralidad ideológicamente asemejaba a una marca fehaciente de salvajismo, a un síntoma irremisible de retraso cultural y mental muy características de esos pueblos enfermos. Desentonaban pues en las "nuevas Republicas". Hacia falta pues remediar aquella tara acudiendo a su exterminación merced a las "guerras de fronteras" (en México, Argentina, en Brasil, por ejemplo), educándola y civilizándola según las pautas de los ideales des Lumières, y sobre todo blanqueando las patrias por unas políticas migratorias oficialmente racistas (como por ejemplo Las leyes de base y colonización adoptadas en Costa Rica en 1862), etc.

Uno de los cometidos de la Revolución cubana, puesto que esta relatoría se basa en el ejemplo de Cuba, era precisamente ir en contra de este orden discursivo eurocéntrica y falocéntrica. Se intentó pues contrarrestar la "cultura burguesa" considerada como una cómplice objetiva de la violencia capitalista e imperial. El fomentar el habla popular, el habla de los sujetos subalternizados, entre los cuales los negros, era entonces parte de la agenda cultural y política de los revolucionarios cubanos.

Pero, ¿efectos que se esperaban del "habla" recobrado del ex- "pieza" o "leña" de Indias en los tiempos revolucionarios cubanos? No es menos legítimo preguntarse si, en el caso del sujeto popular negro, se le daba la palabra porque, a estas alturas, se le consideraba ya mentalmente maduro (no tanto por la edad de los testigos, como en el caso harto conocido de Esteban Montejos en Biografía de un cimarrón, 1967) como para "hablar" (bien, como manda el 
logos y/o la utopia revolucionaria), como para articular un discurso coherente y eficiente o porque, como en los últimos decenios, y fuera del caso cubano, los momentos postmodernos y multiculturales vuelven impostergables la emergencia (consolidación) de la voz del sujeto negro en Américas/Caribes.

Como sea, el fortalecimiento y la institucionalización de la práctica cultural del testimonio ofrece un buen asidero crítico para abordar estas cuestiones de suma importancia puesto que, al fin y al cabo, entroncan con los ideales del "humanismo", de la democracia representativa, con las formas políticas y culturales de representación de un "nosotros" imaginado, fuese regional, nacional o continental (ROSANVALLON, 2014).

Ello tal vez explique que la práctica testimonial desate siempre debates y controversias macanudos, entre universitarios de distintas disciplinas y que no necesariamente comparten las mismas orientaciones epistemológicas y éticas, las mismas percepciones de las prácticas culturales, entre ellos y los políticos, entre los testigos que a veces, como Domitila, acusan a los letrados/acomodados de "negociar" con dolores ajenos, desde un lugar del poder simbólico no necesariamente desligado del orden global del mundo cuyos efectos afectan dramáticamente a los subalternaos.

En este sentido, hablar con o en nombre de los sujetos populares no significa entonces automáticamente solidaridad, alianza política liberadora; antes puede ser percibido como una marca de un paternalismo rampante, una perpetuación de aquella visión colonial y neocolonial que consideraba a los "otros" como menores de edad, incapaces entonces de (auto) representarse. Por ello hacia falta "encauzarlos", llevarlos de la mano en el camino del "hablar bien", del "actuar bien", etc.

Entre las ambivalencias y paradojas que marca la práctica cultural testimonial, remarcaré también que la mayoría de los estudios que versan sobre los testimonios en Américas/Caribes parecen interesarse más en sus contenidos "explícitos" o "inmediatos". Semejante apetencia, sin duda alguna legítima, parece perder de vista las mises en scène fatalmente ligadas al hecho de contar su vida, de contar las horrendas cosas acaecidas la yo/nosotros. Los testimonios publicados no recogen entonces el lenguaje no verbal que esta íntimamente relacionado al hecho de contar: los gestos, los silencios, los visajes, las lágrimas, las risas, las cóleras, etc. Digamos que, en el caso de los sujetos populares negros, los testimonios publica- 
Pontos de Interrogação, v. 3, n. 2, jul./dez. 2013

Revista do Programa de Pós-Graduação em Crítica Cultural

Universidade do Estado da Bahia (UNEB), Campus II - Alagoinhas - BA

dos se muestran incapaces de representar el sygnyfying monkey tan importante en las vivencias y resistencias de los negros en Américas/Caribes.

Por otra, al privilegiar los contenidos se deja de lado la importancia de los (para) textos que abren, cierran o acompañan los testimonios populares: las introducciones o los prefacios de los gestores, de las gestoras, los prefacios de lo-a-s testigo-a-s, las notas al lector, las ilustraciones graficas, los documentos oficiales, los distintos encabezados o exergos, léxicos, notas de pie de páginas (KWEVI-KAYISSA), 1997).

Si bien es cierto que estos (para) textos procuran reforzar y garantizar los efectos de verdad o los efectos reales de las cosas acontecidas que se narran, no denuncian menos las mediaciones del mercado cultural, los horizontes de espera, los hábitos de lecturas que fomentan, consolidan o reproducen. De allí las ambivalencias, paradojas y reducciones que se notan en las diferentes traducciones de los testimonios, empezando por los títulos; de ahí también algunas purgas que sufren los textos testimoniales cuando son publicados por tal o cual casa editorial. ¿Cómo justipreciarlas y a qué se deben?

La misma organización narrativa lineal muchas veces adoptada/privilegiada por los gestores, a la hora de publicar las "voces subalternas" recogidas y decantadas, participa de esos horizontes de espera. Ella introduce un desajuste o una distorsión discursiva entre el momento de enunciación del testigo o de la testiga y el momento de la publicación/lectura de los testimonios que se abre casi siempre por los "orígenes". Como si el hecho de reiterar el roman familial del testigo o de la testiga volviera más apetecible y/o aguantable los horrores que a continuación son narrados. A menos que esta disposición narrativa tenga que ver con el quehacer del etnólogo que primero tiene que establecer a todas costas una empatía con su "informante" para luego abordar más fácilmente los temas a tratar, las memorias a recolectar.

Como sea que fuera, la decisión de mantener este dispositivo, por lo visto, de "laboratorio" en el texto final llama la atención porque al privilegiar este tipo de cronología lineal se corre el riesgo de representar las tragedias padecidas por los testigos como una mera ruptura dentro de una historia personal y natural. ¿Cómo entonces dar cuenta de estos eventos sin quedar atrapado-a en el historicismo siempre al acecho en estos casos?

El desajuste que se observa en la organización narrativa de los testimonios se encuentra también en los textos liminales [como sea el sentido que se le quiera da a "liminalidad" 
(ZOUNGBO, 2007)] que mencioné anteriormente. No es menos llamativo, por ejemplo, que en estos prólogos, el sujeto popular negro deje de ser un "sujeto" (formal) de enunciación para convertirse en un "sujeto"/ "objeto" (real) del quehacer antropológico. De modo que, aunque sea una verdad crítica a gritos, hay que recordarla siempre: los prólogos rebasan la mera función informativa, el contrato de honestidad que se les adscribe o se les reconoce usualmente. En los testimonios populares, no se culpa aquí a los gestores, los prólogos "hablan" más de lo que se cree. La reseña de las condiciones de recopilación/producción de los testimonios que ofrecen sitúan al mismo tiempo el lugar imaginario y disciplinario de producción de esos prólogos. De hecho las reseñas aludidas vienen permeadas, a veces de manera difuminada, por cierto pedagogismo o sea por una lección a cerca de cómo ser buen etnólogo o de cómo hacer buena etnología.

Junto con ello, los prólogos (su dimensión imaginaria) deslindan o inventan una genealogía revolucionaria o anti hegemónica en la cual viene a insertarse, en línea directa, la voz del sujeto testigo negro. El sujeto enunciante (formal) negro pierde así toda posibilidad de control (aunque relativo) de su propio discurso que se encuentra entonces enmarcado por una serie de mediaciones discursivas, políticas e imaginarios.

Me parece que los debates sonados en torno al prólogo de Elizabeth Burgos a la primera edición de Me llamo Rigoberta Menchú y así me nació la conciencia (1982) el prefacio de la misma Domitila a Si me permiten hablar, testimonio de Domitila, una mujer de las minas de Bolivia (1977), con Moema Viezzer como gestora dan cuenta de esas contradicciones. Son notables también en la introducción del propio Miguel Barnet a Biografía de un cimarrón (1968) que tal vez por ser un clásico y celebrado como un modèle du genre (por las ciudades letradas), no haya recibido la atención crítica que merecería. Por otra parte, la nota al lector que abre Reyita, sencillamente. Testimonio de una negra cubana nonagenaria (2010), con Daysi Rubiera Castillo como gestora, desvela aquella genealogía imaginada que yo señalaba.

Estos juicios sumarios, aunque parezcan demasiado estructuralistas y formalistas o literaturizantes, invitan en realidad, lejos de cualquier juicio de valor moral sobre el trabajo de los gestores y fuera de una percepción crítica acartonada muy dada a lamentar la pérdida la "autenticidad" de la voz del sujeto negro popular por las mediaciones que la encierra/enmarcan, a interrogar, insisto en ello, las condiciones de (im) posibilidad de la emergencia y consolida- 
ción de un discurso popular negro en formaciones culturales e ideológicas postcoloniales y postesclavistas (MANGEON, 2010; LOUDE, 2013).

Dicho de otro modo, esas mediaciones o esa "interrupción”, por tomar prestada la palabra al maestro John Beverley (2010), replantean la posibilidad que tiene el sujeto negro en general y popular en particular de "hablar" un lenguaje legible y audible desde aquella faille/impronta, desde lo que yo llamo la "desdicha genealógica" (ZOUNGBO, 2013) que por lo visto sigue marcando profundamente las estructuras imaginarias de representación en las Américas/Caribes, generando y perpetuando una aparente "incomensurabilidad" entre el orden del discurso y la voz popular negra.

Tan es así que, inclusive en la actualidad, lo que se podría considerar como el despunte y/o la consolidación de la voz del sujeto negro en Américas/Caribes sigue lamentablemente siendo percibido, dentro y fuera de las ciudades letradas, dentro y fuera del mercado/campo político o desde la "sociedad civil" como la expresión (dañina cuando no infantil) de revanchismos o resentimientos que por lo demás no tienen cabida considerando las "políticas de identidad" que van teniendo cada vez mas legitimidad y fianza políticas: acciones afirmativas, política de cuotas, reformas constitucionales, reformas en los programas de la educación formal, disposiciones legales y jurídicas anti sexistas, anti racistas, anti homofóbicas, debates en torno a los criterios de censos poblacionales, etc.

Me pregunto entonces si, como se ha venido debatiendo, en parte, desde la publicación de Can The subaltern speak por Gayatri Spivak, lo que define y caracteriza al subalterno negro es el espacio de la ausencia (estructural intrínsecamente ligada al orden de discurso) o si antes (o además de ello) es su effacement onto político e imaginario.

\section{BIBLIOGRAFÍA REFERIDA}

ARONNA, Michael. Testimonial intent and narrative dissonance: the marginal heroes of Miguel Barnet. In: PARAVISINI-GEBERT, Lizabeth and ROMERO-CESAREO, Ivette (eds.), 
Displacements and transformations in caribbean cultures. Gainesville: University Press of Florida, 2008. p.162-187.

BEVERLEY, John. La interrupción del subalterno. University of Pittsburgh: Plural Editores, 2010.

BEVERLEY, John, Achugar Hugo. La voz del otro: testimonio, subalternidad y verdad narrativa. 2nda edición, Ciudad de Guatemala, Revista Abrapalabra, 2002.

HALL, Stuart. What is this «black» in black popular culture in DENT, Gina (ed.). Black Popular culture. Seattle: Bay Press, 1992. p. 21-33.

KWEVI-KAYISSA, Clotilde-Chantal. Le paratextuel et le socio-discursif. Vers une lecture sociocritique de Me llamo Rigoberta Menchú y así me nació la conciencia. Thèse pour le Doctorat Nouveau Régime, Directeur Professeur Edmond Cros. Montpellier, 1997.

LOUDE, Jean-Yves. Pépites brésiliennes. Paris: Actes Sud, 2013.

MANGEON, Anthony. La pensée noire et l'Occident. De la bibliothèque coloniale à Barack Obama. Cabris : Editions Sulliver, 2010.

ROSANVALLON, Pierre. Le parlement des invisibles. Paris: Editions du Seuil-Raconter la vie, 2014.

REDIKER, Marcus. A bord du Négrier. Une histoire atlantique de la traite. Trad. Aurélien Blanchard. Paris: Editions du seuil, 2013.

ZOUNGBO, Victorien Lavou et MARTY, Marlène (éds.). Imaginario racial y construcciones identitarias. Perpignan: Presses Universitaires de Perpignan, 2009.

ZOUNGBO, Victorien Lavou. Outsidering Liminalité des Noir-e-s Amériques/Caraïbes. En hommage à Aimé Césaire. Perpignan: Presses Universitaires de Perpignan, 2007. p.42-62.

ZOUNGBO, Victorien Lavou. Les blancs de l'histoire. Afrodescendance: parcours de représentation et constructions hégémoniques. Perpignan: Presses Universitaires de Perpignan, 2013.

Recebido em: 17 de novembro de 2013.

Aprovado em: 04 de dezembro de 2013. 